\title{
Racial and Ethnic Differences in Falls Among Older Adults: a Systematic Review and Meta-analysis
}

\author{
Natasha Wehner-Hewson $^{1}$ (D) $\cdot$ Paul Watts $^{1}$ (D) $\cdot$ Richard Buscombe $^{1}$ (D) $\cdot$ Nicholas Bourne $^{1} \cdot$ David Hewson $^{2}$ (D)
}

Received: 30 August 2021 / Revised: 21 October 2021 / Accepted: 22 October 2021 / Published online: 16 November 2021

(c) The Author(s) 2021

\begin{abstract}
The aim of this systematic review and meta-analysis was to determine whether differences in reported fall rates exist between different ethnic groups. Searches were carried out on four databases: Medline, Cumulative Index to Nursing and Allied Health Literature (CINAHL), Scopus, and Web of Science. Only English language studies with community-dwelling participants aged $60+$ years were included. Studies also needed to compare fall prevalence for at least two or more ethnic groups. Two reviewers independently screened all articles and evaluated study quality. Twenty-three articles were included for systematic review, and meta-analyses were carried out on the 16 retrospective studies that reported falls in the previous 12 months. The Asian group demonstrated significantly lower fall prevalence than all other ethnic groups at $13.89 \%(10.87,16.91)$. The Hispanic group had a fall prevalence of $18.54 \%$ (12.95, 24.13), closely followed by the Black group at $18.60 \%(13.27,23.93)$. The White group had the highest prevalence at $23.77 \%(18.66,28.88)$. Some studies provided adjusted estimates of effect statistics for the odds/risk of falls, which showed that differences still existed between some ethnic groups even after adjusting for other risk factors. Overall, differences in fall prevalence do appear to exist between different ethnic groups, although the reasons for these differences currently remain undetermined and require further investigation. These findings highlight the need to provide more ethnically tailored responses to public health challenges, which could potentially increase the adherence to prevention interventions, and allow for a more targeted use of resources.
\end{abstract}

Keywords Older adults $\cdot$ Ethnicity $\cdot$ Falls $\cdot$ Prevalence

\section{Introduction}

Falls are one of the most common and most serious problems faced by older adults worldwide [1]. Falls can cause pain, injury and sometimes death, and can also have an impact on mental wellbeing for older adults, their family members and carers [2]. There are wide ranging and severe consequences of falls for both the individual that falls, and for health and care systems [3]. Injuries as the result of a fall range from abrasions and bruises, to hip fracture, with more serious injuries often resulting in institutionalisation [4], while 1 in 5 older adults die within 12 months of a hip fracture [5]. Evidence from systematic reviews of falls

David Hewson

david.hewson@beds.ac.uk

1 School of Health, Sport and Bioscience, University of East London, London, UK

2 Institute for Health Research, University of Bedfordshire, University Square, Luton LU1 3JU, Bedfordshire, UK prevalence in community-based studies shows that the risk of falls is higher for women and with increasing age [6]. However, less is known about differences in the prevalence of falls between ethnic groups.

Substantial health inequalities exist between ethnic groups. In Europe for example, ethnic groups such as South Asians, Black Africans and Black Caribbeans experience higher rates of obesity, diabetes and cardiovascular disease, compared to White Europeans [7, 8]. These inequalities are due to underlying causal factors such as socio-economic factors, including lower levels of education, income, employment and even the built environment, although the contribution each factor plays, and exactly how they interact is difficult to determine. In addition, these inequalities often persist after controlling for socioeconomic disadvantage, suggesting that structural influences such as disparity and discrimination in access to health and social care [9], or cultural differences in behaviours or beliefs may be important factors [10]. 
Health inequalities occur across all age groups, but the greatest differences in health between ethnic groups are among older adults [11]. Health inequalities in older people are likely to increase due to population ageing in countries of all income groups [12]. People are now living for a considerable period in declining health, due to age associated health conditions such as frailty [13]. Falls in particular are likely to increase throughout 'older age' although it is not well understood how ethnic minorities are affected by life course health inequalities as they enter old age $[14,15]$.

The worldwide prevalence of falls is high, commonly reported as being a third for adults aged over sixty-five [16], increasing to $40 \%$ for those over eighty years of age [17]. However, the commonly reported fall prevalence of one-third is usually associated with studies carried out in Western countries, whereas other countries have reported differences in fall prevalence. For instance, China and Japan have noticeably lower reported fall rates than those seen in the West. A systematic review by Kwan et al. [18] reported a median fall prevalence of $18 \%$ in Chinese people from a sample of 21 studies. However, there have been very few studies looking at fall rates in pluricultural populations. Different ethnic groups within a country share common local cultural factors, while potentially differing in specific factors related to ethnicity. For example, within a community, obesity may be more prevalent in a particular ethnic group, even though all members of the community can be expected to be exposed to the same public health messaging about its risks via various media. This may be due to cultural attitudes to physical activity, food preferences, and body image [19].

This is particularly true for migrant groups [20]. In addition, studies that directly compare ethnic groups provide a homogenous methodology to each group, rather than different studies, using different methodologies looking at single ethnicities. The aim of this systematic review is therefore to determine whether differences in reported fall rates exist between different ethnic groups.

\section{Methods}

\section{Search Strategy}

The search was performed and reported following the Preferred Reporting Items for Systematic reviews and MetaAnalyses (PRISMA) [21]. Searches were carried out on the following databases: Medline, Cumulative Index to Nursing and Allied Health Literature (CINAHL), Scopus and Web of Science. Other relevant studies were also identified following individual searches of the reference lists in the articles selected. There was no limitation in publication date, and any articles that satisfied the search criteria were selected, up to the date of search, the end of December 2020. The Cochrane Population, Intervention, Comparison, Outcome (PICO) methodology was used to determine the keywords to be used in the search [22]. A summary of the PICO search strategy is shown in Table 1.

\section{Selection Criteria}

This review included studies of community-dwelling participants, while studies including institutionalised people (hospitals, care homes...) were excluded. All participants were aged $60+$ years, and any studies including younger participants were excluded. To be included, studies needed to provide results separately either for all ethnic groups in the same country, or the same ethnic group in multiple countries. Studies where ethnic identity was not specified, contained mixed ethnic groups, groups titled 'other', or had only single ethnic groups with no comparison to others, were excluded. Studies needed to report fall prevalence, either as

Table 1 PICO Search keywords and MeSH terms

\begin{tabular}{|c|c|c|c|}
\hline PICO Term & Description & Keywords/MeSH & Search location \\
\hline \multirow[t]{4}{*}{ P-Population } & \multirow[t]{2}{*}{ Participants aged $60+$} & Elder* OR older & Title/Abstract \\
\hline & & Aged & MeSH heading \\
\hline & Community-dwelling & - & \\
\hline & Ethnically or culturally homogenous population & Ethni* OR culture* OR rac* & Title/Abstract \\
\hline I-Intervention & None & - & N/A \\
\hline C-Comparison & $\begin{array}{l}\text { Studies must include a comparison between two or more } \\
\text { ethnic/cultural/racial groups }\end{array}$ & - & N/A \\
\hline \multirow[t]{3}{*}{ O-Outcome(s) } & \multirow[t]{2}{*}{ Primary: fall prevalence } & Fall* & Title/Abstract \\
\hline & & Fall & MeSH heading \\
\hline & Secondary: Fall with injury prevalence & - & N/A \\
\hline T-Time & Unlimited & - & N/A \\
\hline S-Study design & Any quantitative study & - & N/A \\
\hline
\end{tabular}


number of falls, rate of falls or number of participants who experienced at least one fall, to be included. Only studies written in English were included.

\section{Data Extraction}

Keyword searches were carried out on all four databases. The results were imported into EndNote X9 (Clarivate Analytics, Philadelphia, PA, USA), and all duplicates were removed. Titles and abstracts were reviewed by two researchers to determine relevant studies. Full text versions of each paper were obtained for detailed review and extraction of data. Selected data from each study were entered on an Excel template, with extracted data including participant demographics such as age, ethnicity, country of study, living situation, whether the group was ethnically homogeneous, comparison of two or more ethnic groups, fall prevalence and study design. Selected studies were critically assessed using the 'Quality Assessment Tool for Observational Cohort and Cross-Sectional Studies' [23]. Fourteen questions were answered as 'yes', 'no' or 'Other (cannot be determined, not applicable, not reported)'. Two reviewers assessed all articles independently, and any disagreements were resolved following discussion with a third party. A score was generated as a percentage, without considering any 'not applicable' responses. Scores rated $<50 \%$ were considered to be 'poor', with $50-74 \%$ considered to be 'fair', while those rated $\geq 75 \%$ considered to be of 'good' quality.

\section{Meta-Analysis}

Following the systematic review, quantitative meta-analysis was carried out in order to provide an overall fall prevalence for the largest groups present in the literature. The different ethnic groups were combined, where possible, under four general headings: Asian (including Asian, Chinese, Filipino and Japanese), Black (including African-American, AfroCaribbean, Black, and Black-African), Hispanic (including Latino and Hispanic) and White (including Australian-born Australian, Caucasian, European-American, Italian-born Australian and Non-Hispanic White). These groups were chosen based on the NIH definitions for racial and ethnic categories [24].

The heterogeneity of the selected studies was evaluated using the $I^{2}$ statistic, with boundaries of 25\%,50\% and $75 \%$ taken to represent low, moderate and high heterogeneity, respectively [25]. Due to the high heterogeneity found across the studies with a fixed model, a random effects model was used for all meta-analyses. The meta-analysis was performed using a Microsoft Excel spreadsheet adapted from Neyeloff et al. [26]. Fall prevalence rates were weighted across ethnic groups using the inverse variance for each study. Data were reported as mean prevalence rates and $95 \%$ confidence intervals, with statistical significance taken to be $p<0.05$. Forest plots were used to visualize the distribution of the fall prevalence data from the different studies included.

\section{Results}

\section{Article Selection}

The article selection PRISMA flowchart for this systematic review is included in Fig. 1. A total of 9653 articles was identified during the database searches, which decreased to 6339 following removal of duplicates. After title and abstract screening, 6272 articles were removed leaving 67 articles for full-text appraisal. A further 44 articles were excluded due to reasons including lack of ethnic comparison, the inclusion of participants under the age of 60, non-English language articles, ethnic groups that were not homogeneous or participants who were not community-dwelling. The final selection consisted of 23 articles, the characteristics of which are shown in Table 2, including quality appraisal scores.

\section{Article Description}

The selected articles included 5,727,024 participants overall, with study sample sizes ranging from 114 [43] to 5,519,341 [45]. Studies were conducted with many different ethnic groups in several countries. There were 13 studies in the USA; two studies in Australia, Japan, Malaysia and Singapore; and 1 study from Brazil, Hong Kong, The Netherlands, South Africa and Taiwan. The 23 articles included nineteen retrospective studies, three prospective studies and one Electronic Medical Record study. Of the retrospective studies, 16 reported falls in the previous 12 months, two reported falls in the previous 24 months, while one study looked at falls in the previous 3 months.

\section{Quality Assessment}

The quality appraisal scores ranged from 60 to $100 \%$ of the maximum score for each article. Of the 23 studies included, 6 were rated as fair, with the remaining 17 articles rated as good.

\section{Fall Prevalence}

Fall prevalence was reported for 22 of the 23 studies and is shown in Table 3. Prevalence varied widely across the studies, from $2.9 \%$ (95\% CI: 0.1, 5.6) for Chinese people in Malaysia [48], to $44.5 \%$ (95\% CI: 37.8, 51.2) for Malays in Malaysia [39]. 
Fig. 1 PRISMA flowchart of the article selection process [21]
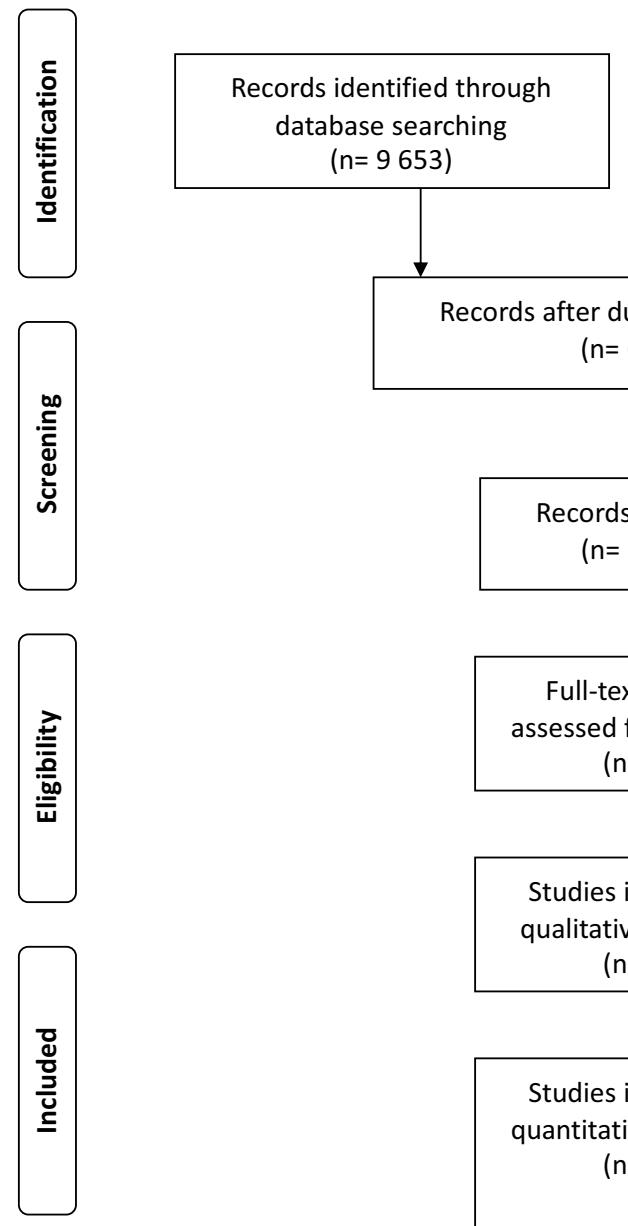

$(n=9653)$
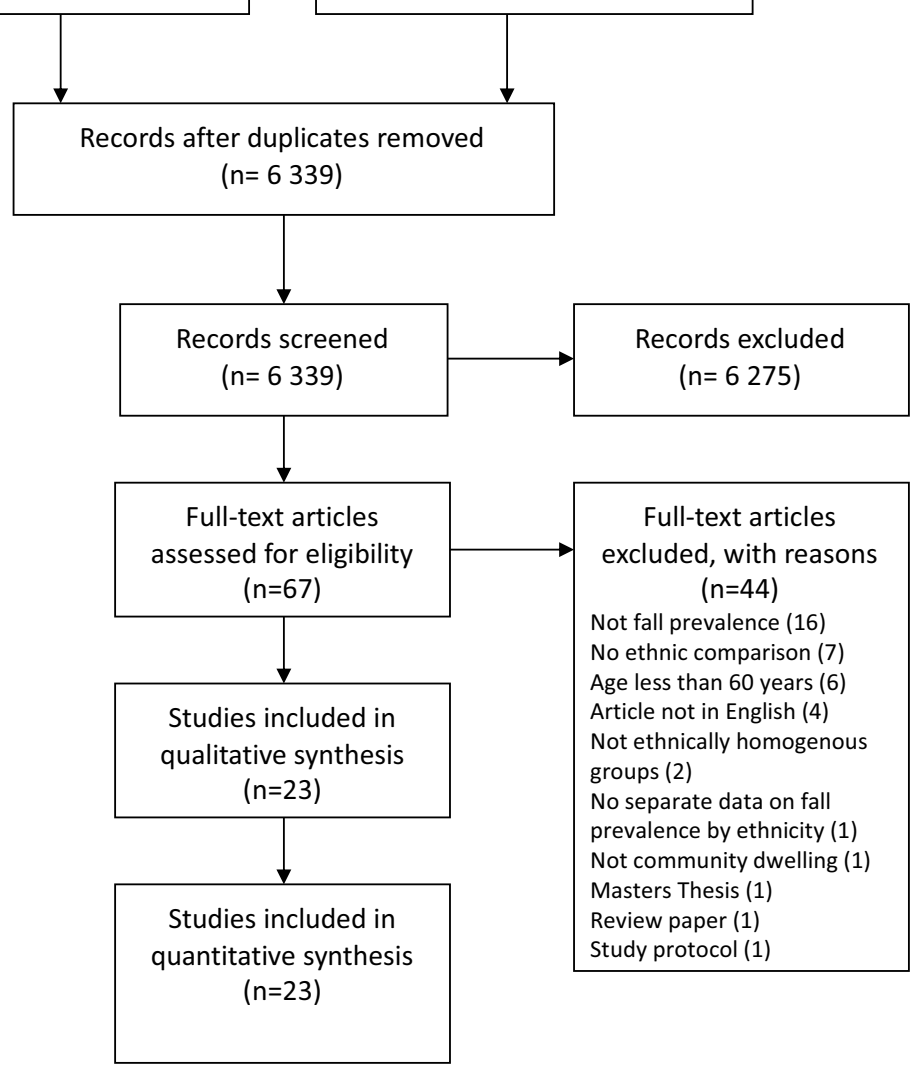

A meta-analysis of fall prevalence was undertaken only for those 16 retrospective studies that reported falls in the previous 12 months, with Forest Plots shown in Figs. 2, 3, 4 and 5.

The Asian group demonstrated significantly lower fall prevalence than all other ethnic groups at $13.89 \%(10.87,16.91)$. The Hispanic group had a fall prevalence of 18.54\% (12.95, 24.13), closely followed by the Black group at $18.60 \%(13.27,23.93)$. The White group had the highest prevalence at $23.77 \%$ (18.66, 28.88). Heterogeneity of studies included in the meta-analysis was low for the Black, and White groups, with $I^{2}$ veasures of 17.57, and 18.96 respectively. It was moderate for the Asian group at 32.02, and high for the Hispanic group at 55.49.

\section{Fall Risk}

\section{Unadjusted Odds Ratios/Relative Risk}

Most studies included comparisons with white participants (seven studies in the USA, one in Australia and one in South Africa), with only a few comparing fall prevalence with other ethnic groups. The unadjusted effect statistics of these comparisons for single falls are shown in Table 4. Overall results followed those of the fall prevalence meta-analysis, suggesting that White older adults tend to fall more than other ethnic groups (Black, Asian, Hispanic, Caribbean, Japanese, Filipino). There was some evidence of other differences in Asian countries, but the results were variable.

\section{Adjusted Odds Ratios/Relative Risk}

Some studies provided adjusted estimates of effect statistics for the odds/risk of falls. These adjustments included a range of factors such as co-morbidities, depression, mobility limitations, functional tests and sociodemographic characteristics. These adjusted effect statistics are shown for single falls in Table 5, and recurrent falls in Table 6.

These data show differences in the odds/risk of falling still existed between some ethnic groups even after adjusting for other risk factors. For single falls, seven of the eight studies reported a statistically significant difference in the risk of falls between ethnic groups, generally showing the White people tend to fall more than Black and Asian older adults, but did not differ from Hispanics. When observing differences in recurrent falls for the two studies in which this was reported, there was again a reduced risk of falling observed for Asian older adults compared to White in the study of Kwan et al. [38]. 
Table 2 Characteristics of selected articles

\begin{tabular}{|c|c|c|c|c|c|}
\hline Authors & Country & Ethnic group & Age & Fall reporting & Quality score (\%) \\
\hline \multirow[t]{2}{*}{ Aoyagi et al. (1998) [27] } & Japan & Japanese & $\geq 6$ years & Retrospective 12 months & 60.0 \\
\hline & USA & Japanese & & & \\
\hline Chan et al. (1997) [28] & Singapore & Chinese, Indian, Malay & $\geq 60$ years & Retrospective 12 months & 60.0 \\
\hline Chen et al. (2018) [29] & Singapore & Chinese, Indian, Malay & $\geq 65$ years & Retrospective 12 months & 87.5 \\
\hline \multirow[t]{2}{*}{ Davis et al. (1999) [30] } & USA mainland & White & $\geq 65$ years & Prospective 24 months & 90.0 \\
\hline & Hawaii & Japanese & $\geq 65$ years & & \\
\hline $\begin{array}{l}\text { de Rekeneire et al. (2003) } \\
\text { [31] }\end{array}$ & USA & Black, White & $70-79$ years & Retrospective 12 months & 87.5 \\
\hline El Fakiri et al. (2018) [32] & The Netherlands & $\begin{array}{l}\text { White, Moroccan, Suri- } \\
\text { namese, Turkish }\end{array}$ & $\geq 65$ years & Retrospective 12 months & 70.0 \\
\hline Faulkner et al. (2005) [33] & USA & Black, White & $\geq 65$ years & $\begin{array}{l}\text { Prospective every } 4 \text { months } \\
\text { for up to } 5.7 \text { years }\end{array}$ & 88.9 \\
\hline Geng et al. (2017) [34] & USA & $\begin{array}{l}\text { Asian, Black, Hispanic, } \\
\text { White }\end{array}$ & $65-90$ years & Retrospective 12 months & 80.0 \\
\hline Hanlon et al. (2002) [35] & USA & Black, White & $\geq 65$ years & Retrospective 12 months & 90.0 \\
\hline Kalula et al. (2015) [36] & South Africa & Black African, White & $\geq 65$ years & Retrospective 12 months & 72.7 \\
\hline Karter et al. (2015) [37] & USA & $\begin{array}{l}\text { Asian, Black, Filipino, His- } \\
\text { panic, White }\end{array}$ & $\geq 60$ years & EMR data only & 100.0 \\
\hline \multirow[t]{4}{*}{ Kwan et al. (2013) [38] } & Hong Kong & Chinese & $\geq 65$ years & Prospective 12 months & 100.0 \\
\hline & Taiwan & Chinese & & Prospective 24 months & \\
\hline & Australia & Chinese & & Prospective 12 months & \\
\hline & Australia & White & & Prospective 12 months & \\
\hline Kwon et al. (2018) & USA & $\begin{array}{l}\text { Asian, Black, Hispanic, } \\
\text { White }\end{array}$ & $\geq 65$ years & Retrospective 12 months & 77.8 \\
\hline $\begin{array}{l}\text { Leong Joyce et al. (2020) } \\
\text { [39] }\end{array}$ & Malaysia & Chinese, Indian, Malay & $\geq 60$ years & Retrospective 12 months & 80.0 \\
\hline Means et al. (2000) [40] & USA & Black, White & $\geq 65$ years & Retrospective 12 months & 66.7 \\
\hline $\begin{array}{l}\text { Nicklett and Taylor (2014) } \\
\text { [41] }\end{array}$ & USA & Black, Hispanic, White & $\geq 65$ years & Retrospective 24 months & 90.0 \\
\hline $\begin{array}{l}\text { Qin and Baccaglini (2016) } \\
\text { [42] }\end{array}$ & USA & $\begin{array}{l}\text { Asian, Black, Hispanic, } \\
\text { White }\end{array}$ & $\geq 65$ years & Retrospective 12 months & 87.5 \\
\hline \multirow[t]{2}{*}{ Sampaio et al. (2013) [43] } & Brazil & Brazilian & $\geq 65$ years & Retrospective 12 months & 70.0 \\
\hline & Japan & Japanese & & & \\
\hline Stanaway et al. (2011) [44] & Australia & $\begin{array}{l}\text { Australian-born Australian, } \\
\text { Italian-born Australian }\end{array}$ & $\geq 70$ years & $\begin{array}{l}\text { Retrospective } 12 \text { months, fol- } \\
\text { lowed by prospective every } \\
4 \text { months for } 4-40 \text { months }\end{array}$ & 90.9 \\
\hline Stevens et al. (2008) [45] & USA & $\begin{array}{l}\text { American Indian/Alaskan } \\
\text { Native, Black, Hispanic, } \\
\text { White }\end{array}$ & $\geq 65$ years & Estimated data & 75.0 \\
\hline Sun et al. (2016) [46] & USA & Black, White & $\geq 65$ years & Retrospective 12 months & 90.9 \\
\hline Vieira et al. (2015) [47] & USA & $\begin{array}{l}\text { African-American, Afro- } \\
\text { Caribbean, European- } \\
\text { American, Hispanic }\end{array}$ & $\geq 60$ years & Retrospective 24 months & 77.8 \\
\hline Yeong et al. (2016) [48] & Malaysia & $\begin{array}{l}\text { Chinese, Indian, Malay, } \\
\text { Indigenous }\end{array}$ & $\geq 60$ years & Retrospective 12 months & 77.8 \\
\hline
\end{tabular}

\section{Discussion}

This systematic review was limited to only those studies in which fall prevalence was compared between two or more ethnic groups in an attempt to increase the heterogeneity of study design. Studies in which fall prevalence was only reported for a single ethnic group were excluded. However, the wide range of countries in which the studies were carried 
Table 3 Prevalence of falls

\begin{tabular}{|c|c|c|c|c|c|c|}
\hline Authors & Country & Ethnic group & Sample size & Type of fall & $\begin{array}{l}\text { Fall preva- } \\
\text { lence }(\%)\end{array}$ & $\begin{array}{l}95 \% \\
\text { Confidence } \\
\text { Interval }\end{array}$ \\
\hline \multirow[t]{4}{*}{ Aoyagi et al. (1998) [27] } & Japan & Japanese men & 624 & Single fall & $9.5 \%$ & $(7.2,11.8)$ \\
\hline & Japan & Japanese-American men & 436 & Single fall & $11.5 \%$ & $(8.4,14.5)$ \\
\hline & USA & Japanese-American women & 618 & Single fall & $16.8 \%$ & $(13.9,19.8)$ \\
\hline & USA & Japanese women & 910 & Single fall & $19.1 \%$ & $(16.6,21.7)$ \\
\hline \multirow[t]{3}{*}{ Chan et al. (1997) [28] } & Singapore & Indian & 24 & Single fall & $4.2 \%$ & $(0.0,12.2)$ \\
\hline & Singapore & Chinese & 333 & Single fall & $17.1 \%$ & $(13.1,21.2)$ \\
\hline & Singapore & Malay & 31 & Single fall & $35.5 \%$ & $(18.6,52.3)$ \\
\hline \multirow[t]{6}{*}{ Chen et al. (2018) [29] } & Singapore & Malay & 327 & Injurious & $4.6 \%$ & $(2.3,6.9)$ \\
\hline & Singapore & Chinese & 1446 & Injurious & $4.8 \%$ & $93.7,5.9)$ \\
\hline & Singapore & Indian & 202 & Injurious & $6.4 \%$ & $(3.1,9.8)$ \\
\hline & Singapore & Chinese & 1446 & Single fall & $11.7 \%$ & $(10.0,13.3)$ \\
\hline & Singapore & Malay & 327 & Single fall & $17.4 \%$ & $(13.3,21.5)$ \\
\hline & Singapore & Indian & 202 & Single fall & $20.8 \%$ & $(15.2,26.4)$ \\
\hline \multirow[t]{2}{*}{ de Rekeneire et al. (2003) [31] } & USA & Black & 1270 & Single fall & $18.8 \%$ & $(16.7,21.0)$ \\
\hline & USA & White & 1780 & Single fall & $23.2 \%$ & $(21.2,25.2)$ \\
\hline \multirow[t]{8}{*}{ El Fakiri et al. (2018) [32] } & The Netherlands & White & 7952 & Recurrent falls & $13.1 \%$ & $(12.4,13.9)$ \\
\hline & The Netherlands & Moroccan & 165 & Recurrent falls & $17.0 \%$ & $(11.2,22.7)$ \\
\hline & The Netherlands & Surinamese & 587 & Recurrent falls & $21.0 \%$ & $(17.7,24.2)$ \\
\hline & The Netherlands & Moroccan & 165 & Single fall & $30.3 \%$ & $(23.3,37.3)$ \\
\hline & The Netherlands & Turkish & 188 & Recurrent falls & $20.7 \%$ & $(14.9,26.5)$ \\
\hline & The Netherlands & White & 7952 & Single fall & $32.5 \%$ & $(31.5,33.5)$ \\
\hline & The Netherlands & Surinamese & 587 & Single fall & $37.1 \%$ & $(33.2,41.0)$ \\
\hline & The Netherlands & Turkish & 188 & Single fall & $32.4 \%$ & $(25.8,39.1)$ \\
\hline \multirow[t]{2}{*}{ Faulkner et al. (2005) [33] } & USA & Caucasian & 1665 & Single fall & $24.7 \%$ & $(22.6,26.8)$ \\
\hline & USA & Black & 156 & Single fall & $27.6 \%$ & $(20.6,34.6)$ \\
\hline \multirow[t]{4}{*}{ Geng et al. (2017) [34] } & USA & Asian & 684 & Single fall & $20.0 \%$ & $(17.0,23.0)$ \\
\hline & USA & Black & 463 & Single fall & $23.3 \%$ & $(19.5,27.2)$ \\
\hline & USA & Hispanic & 425 & Single fall & $27.8 \%$ & $(23.5,32.0)$ \\
\hline & USA & White & 4705 & Single fall & $28.5 \%$ & $(27.2,29.8)$ \\
\hline \multirow[t]{2}{*}{ Hanlon et al. (2002) [35] } & USA & Black & 1049 & Single fall & $20.2 \%$ & $(17.8,22.6)$ \\
\hline & USA & White & 1947 & Single fall & $23.2 \%$ & $(21.3,25.1)$ \\
\hline \multirow[t]{2}{*}{ Kalula et al. (2015) [36] } & South Africa & Black African & 283 & Single fall & $6.4 \%$ & $(0.0,14.6)$ \\
\hline & South Africa & White & 140 & Single fall & $42.9 \%$ & $(40.0,45.7)$ \\
\hline \multirow[t]{5}{*}{ Karter et al. (2015) [37] } & USA & Filipino & 8162 & Single fall & $3.7 \%$ & $(3.3,4.1)$ \\
\hline & USA & Asian & 11,275 & Single fall & $5.3 \%$ & $(4.9,5.7)$ \\
\hline & USA & Black & 11,417 & Single fall & $5.7 \%$ & $(5.3,6.2)$ \\
\hline & USA & Latino & 14,324 & Single fall & $6.8 \%$ & $(6.4,7.2)$ \\
\hline & USA & Non-Hispanic White & 63,509 & Single fall & $8.5 \%$ & $(8.3,8.7)$ \\
\hline \multirow[t]{4}{*}{ Kwan et al. (2013) [38] } & Hong Kong & Chinese & 201 & Single fall & $26.4 \%$ & $(21.2,31.5)$ \\
\hline & Taiwan & Chinese & 280 & Single fall & $28.9 \%$ & $(22.8,35.0)$ \\
\hline & Australia & Chinese & 211 & Single fall & $28.9 \%$ & $(22.8,35.0)$ \\
\hline & Australia & White & 764 & Single fall & $32.1 \%$ & $(29.4,34.7)$ \\
\hline \multirow[t]{4}{*}{ Kwon et al. (2018) } & USA & Asian & 1199 & Recurrent falls & $7.6 \%$ & $(6.1,9.1)$ \\
\hline & USA & White & 10,527 & Recurrent falls & $12.8 \%$ & $(12.2,13.4)$ \\
\hline & USA & Hispanic & 1423 & Recurrent falls & $14.8 \%$ & $(13.0,16.7)$ \\
\hline & USA & Black & 595 & Recurrent falls & $14.1 \%$ & $(11.3,16.9)$ \\
\hline
\end{tabular}


Table 3 (continued)

\begin{tabular}{|c|c|c|c|c|c|c|}
\hline Authors & Country & Ethnic group & Sample size & Type of fall & $\begin{array}{l}\text { Fall preva- } \\
\text { lence }(\%)\end{array}$ & $\begin{array}{l}95 \% \\
\text { Confidence } \\
\text { Interval }\end{array}$ \\
\hline \multirow[t]{3}{*}{ Leong Joyce et al. (2020) [39] } & Malaysia & Malay & 209 & Single fall & $44.5 \%$ & $(37.8,51.2)$ \\
\hline & Malaysia & Chinese & 49 & Single fall & $34.7 \%$ & $(21.4,48.0)$ \\
\hline & Malaysia & Indian & 50 & Single fall & $14.0 \%$ & $(4.4,23.6)$ \\
\hline \multirow[t]{2}{*}{ Means et al. (2000) [40] } & USA & Black & 118 & Single fall & $32.2 \%$ & $(23.8,40.6)$ \\
\hline & USA & White & 180 & Single fall & $32.8 \%$ & $(25.9,39.6)$ \\
\hline \multirow[t]{3}{*}{ Nicklett and Taylor (2014) [41] } & USA & Black & 1326 & Single fall & $26.8 \%$ & $(24.4,29.2)$ \\
\hline & USA & White & 8429 & Single fall & $29.2 \%$ & $(28.2,30.2)$ \\
\hline & USA & Hispanic & 729 & Single fall & $31.6 \%$ & $(28.2,34.9)$ \\
\hline \multirow[t]{4}{*}{ Qin and Baccaglini (2016) [42] } & USA & Black & 583 & Recurrent falls & $9.8 \%$ & $(7.4,12.2)$ \\
\hline & USA & Asian & 1193 & Recurrent falls & $10.1 \%$ & $(8.4,11.9)$ \\
\hline & USA & White & 10,359 & Recurrent falls & $13.0 \%$ & $(12.3,13.6)$ \\
\hline & USA & Hispanic & 1395 & Recurrent falls & $14.3 \%$ & $(12.5,16.2)$ \\
\hline \multirow[t]{2}{*}{ Sampaio et al. (2013) [43] } & Brazil & Brazilian & 74 & Single fall & $27.0 \%$ & $(16.9,37.1)$ \\
\hline & Japan & Japanese & 40 & Single fall & $32.5 \%$ & $(18.0,47.0)$ \\
\hline \multirow[t]{2}{*}{ Stanaway et al. (2011) [44] } & Australia & Italian-born Australian & 335 & Recurrent falls & $11.3 \%$ & $(7.9,14.7)$ \\
\hline & Australia & Australian-born Australian & 848 & Recurrent falls & $22.4 \%$ & $(19.6,25.2)$ \\
\hline \multirow[t]{5}{*}{ Stevens et al. (2008) [45] } & USA & Black & 346,155 & Single fall & $13.0 \%$ & $(12.9,13.1)$ \\
\hline & USA & White & $4,643,692$ & Single fall & $15.8 \%$ & $(15.8,15.8)$ \\
\hline & USA & Hispanic & 457,096 & Single fall & $17.4 \%$ & $(17.3,17.5)$ \\
\hline & & American Indian/Alaskan & & & & \\
\hline & USA & Native & 72,398 & Single fall & $27.8 \%$ & $(27.5,28.1)$ \\
\hline \multirow[t]{2}{*}{ Sun et al. (2016) [46] } & USA & Black & 1662 & Single fall & $27.1 \%$ & $(24.9,29.2)$ \\
\hline & USA & White & 5186 & Single fall & $33.8 \%$ & $(32.5,35.1)$ \\
\hline \multirow[t]{4}{*}{ Vieira et al. (2015) [47] } & USA & Afro-Caribbean & 109 & Single fall & $23.9 \%$ & $(15.9,31.9)$ \\
\hline & USA & European-American & 222 & Single fall & $38.7 \%$ & $(32.3,45.1)$ \\
\hline & USA & Hispanic & 113 & Single fall & $38.9 \%$ & $(29.9,47.9)$ \\
\hline & USA & African-American & 106 & Single fall & $39.6 \%$ & $(30.3,48.9)$ \\
\hline \multirow[t]{3}{*}{ Yeong et al. (2016) [48] } & Malaysia & Chinese & 140 & Single fall & $2.9 \%$ & $(0.1,5.6)$ \\
\hline & Malaysia & Indian & 28 & Single fall & $3.6 \%$ & $(0.0,10.4)$ \\
\hline & Malaysia & Malay & 631 & Single fall & $4.1 \%$ & $(2.6,5.7)$ \\
\hline
\end{tabular}

out, the ethnic groups observed and the differing methodologies used all gave substantial variability to the data.

This variability is evident in the wide range of fall prevalence reported, which ranged from 2.9 to $44.5 \%$. In order to synthesise the data from these multiple studies, a metaanalysis was carried out, using a random-effects model due to the variability of the data. This analysis showed that differences were apparent between the reported fall rates of Asian, Hispanic, Black and White populations, listed here from lowest to highest fall prevalence. This observation was confirmed by unadjusted measures of fall risk, which suggested that White people tend to fall more than other ethnic groups. Even when adjusted for a wide range of contributing factors, White populations had a higher risk of falling than other ethnic groups, both for single and recurrent falls. This is an interesting finding, as the majority of these studies were in the USA where African-American populations have poorer health and living conditions than White Americans in the same area [49], and yet when their risk of falling was adjusted for these inequalities, it was still lower than that for the White older adults. This is also contrary to other age-related conditions such as frailty, in which higher rates of frailty have been reported for African Americans in the USA [50, 51].

There are many potential reasons for the differences observed in these studies. It has been shown that there may be a difference in attitudes to fall risk and participation in risk-taking behaviours between Asian and White groups [38]. Lower fall rates in Chinese groups may be due to greater fear of falling as evidenced by their higher scores in FES-I tests, as well as different cultural behaviours such as greater use of walking sticks. These two factors could 
Fig. 2 Fall prevalence for Asian ethnicity $\left(I^{2} v=32.02, p<0.001\right.$, $Q v=22.07)$

\section{Article}

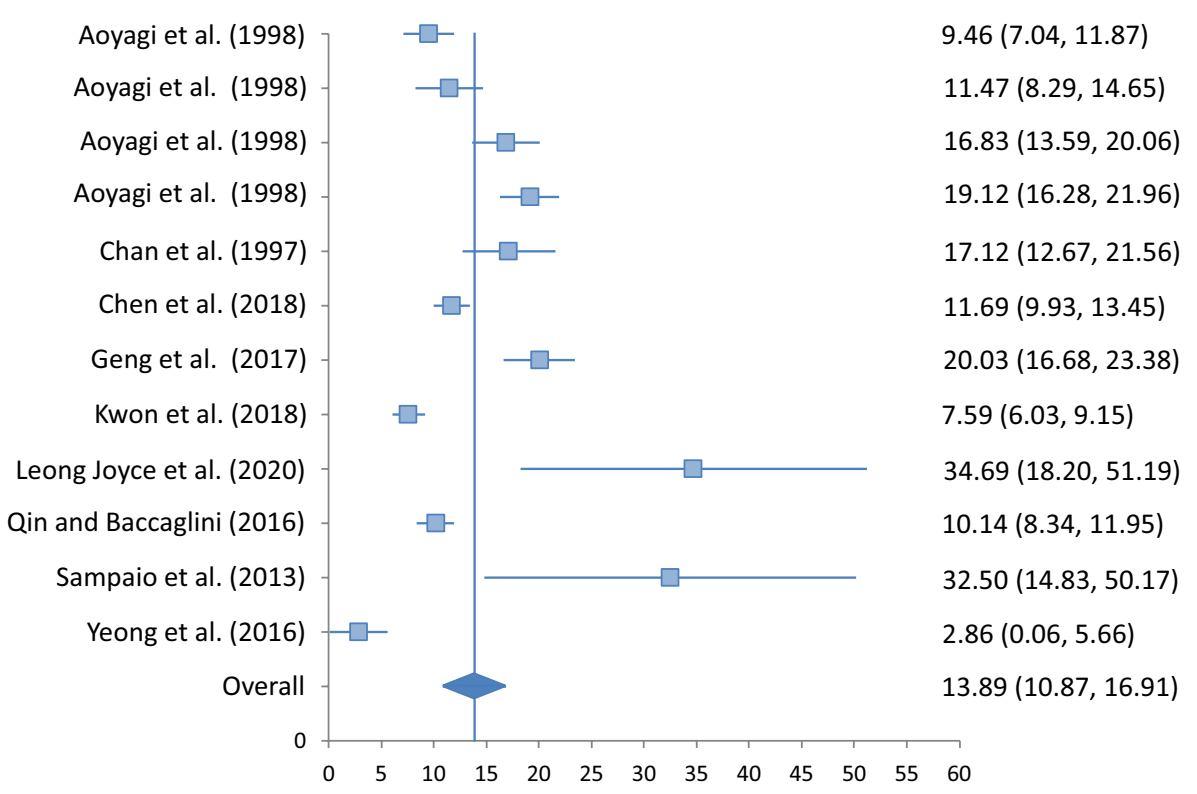

Fall prevalence $\pm 95 \% \mathrm{Cl}$

$46(7.04,11.87)$

$16.83(13.59,20.06)$

$19.12(16.28,21.96)$

21.56)

$20.03(16.68,23.38)$

$.59(6.03,9.15)$

$4.69(18.20,51.19)$

$2.86(0.06,5.66)$

$13.89(10.87,16.91)$
Fig. 3 Fall prevalence for Black ethnicity $\left(I^{2} v=17.57, p<0.001\right.$, $Q v=8.49)$

\section{Article}

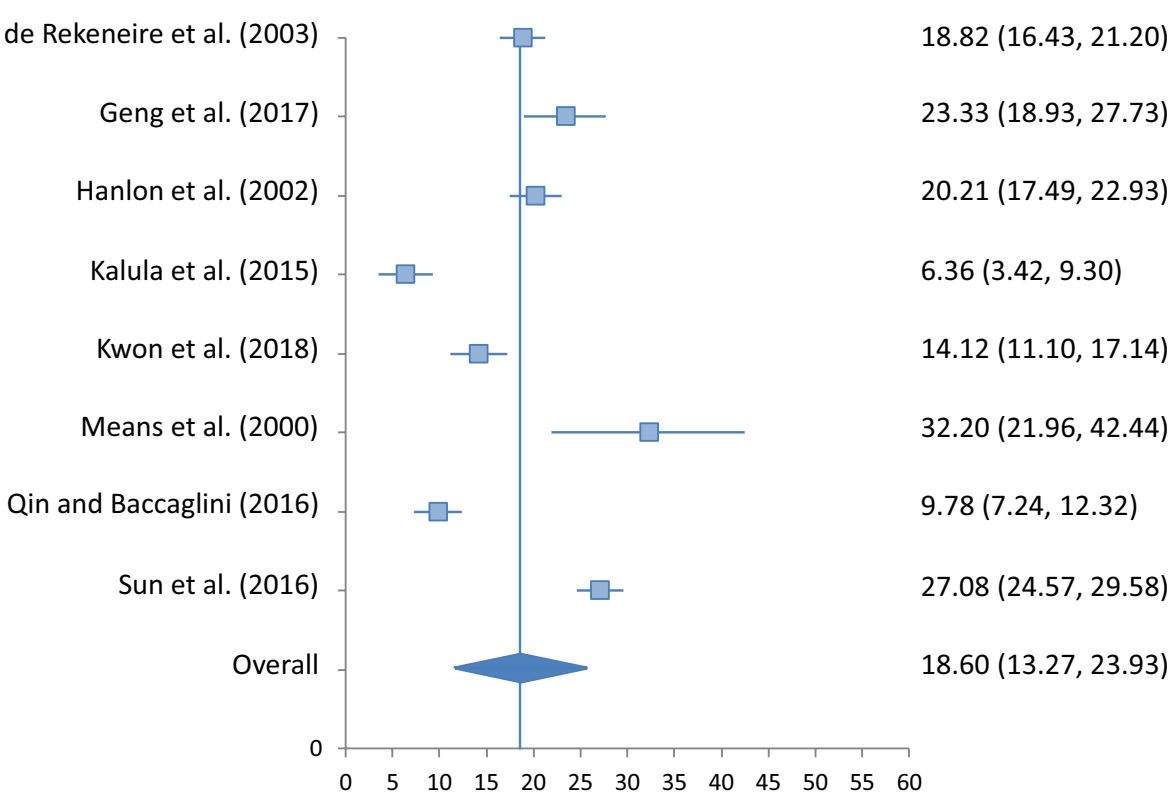

result in lower levels of risk-taking behaviours. In addition, increasing fall prevalence with increasing age may affect results in different countries and ethnic groups due to differences in local life expectancy.

In reality, differences in fall prevalence are probably due to a complex interaction of factors including culturally specific behaviours and beliefs, general health characteristics and sociodemographic elements. Culturally specific behaviours may include differences such as those who wish to avoid losing face or showing weakness associated with older age [52], compared with those who are more willing to accept assistance [38]. Health beliefs could involve issues such as having a fatalistic attitude towards falls and potential prevention interventions [53,54]. Health issues may include chronic illnesses, functional impairments including visual problems or walking difficulties, or common geriatric conditions such as cognitive impairments [52]. BMI is also a risk factor for falls as those with high BMI measures often 
Fig. 4 Fall prevalence for Hispanic ethnicity $\left(I^{2} v=55.49\right.$, $p<0.001, Q v=4.49$ )

Article

Fall prevalence $\pm 95 \% \mathrm{Cl}$

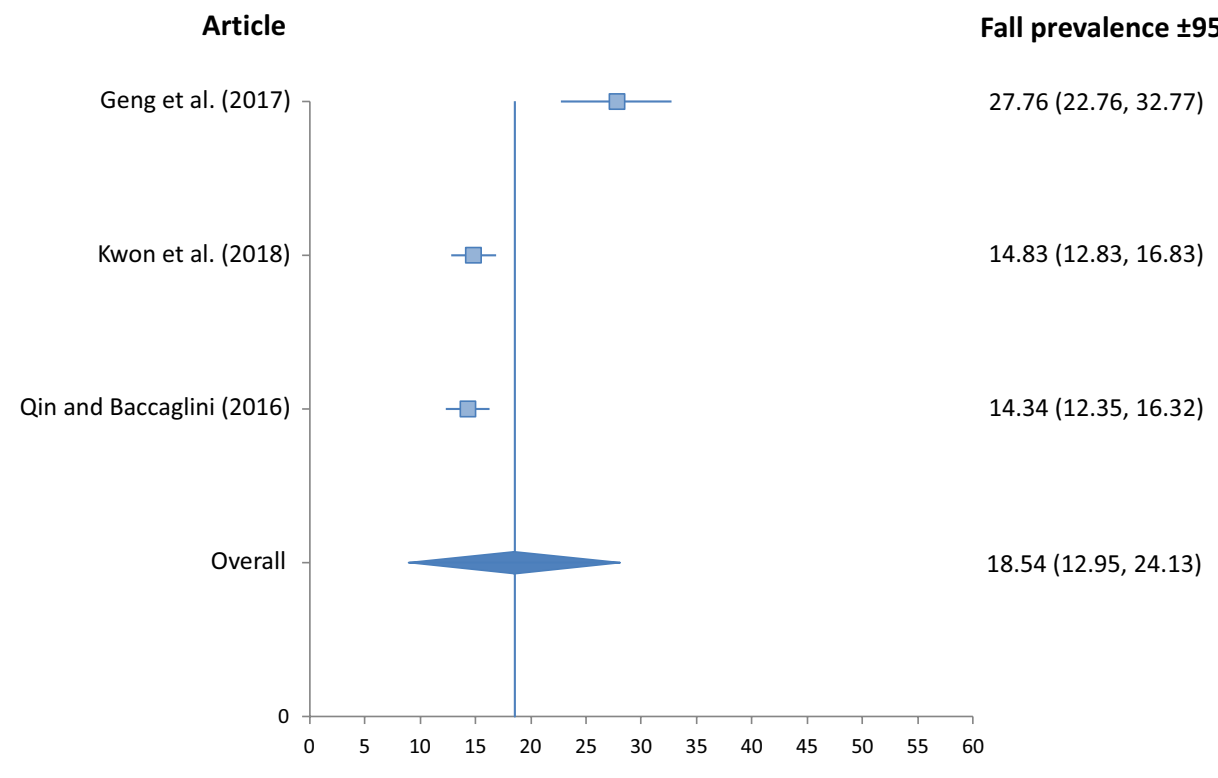

Article

Fig. 5 Fall prevalence for White ethnicity $\left(I^{2} v=18.96, p<0.001\right.$, $Q v=13.57)$

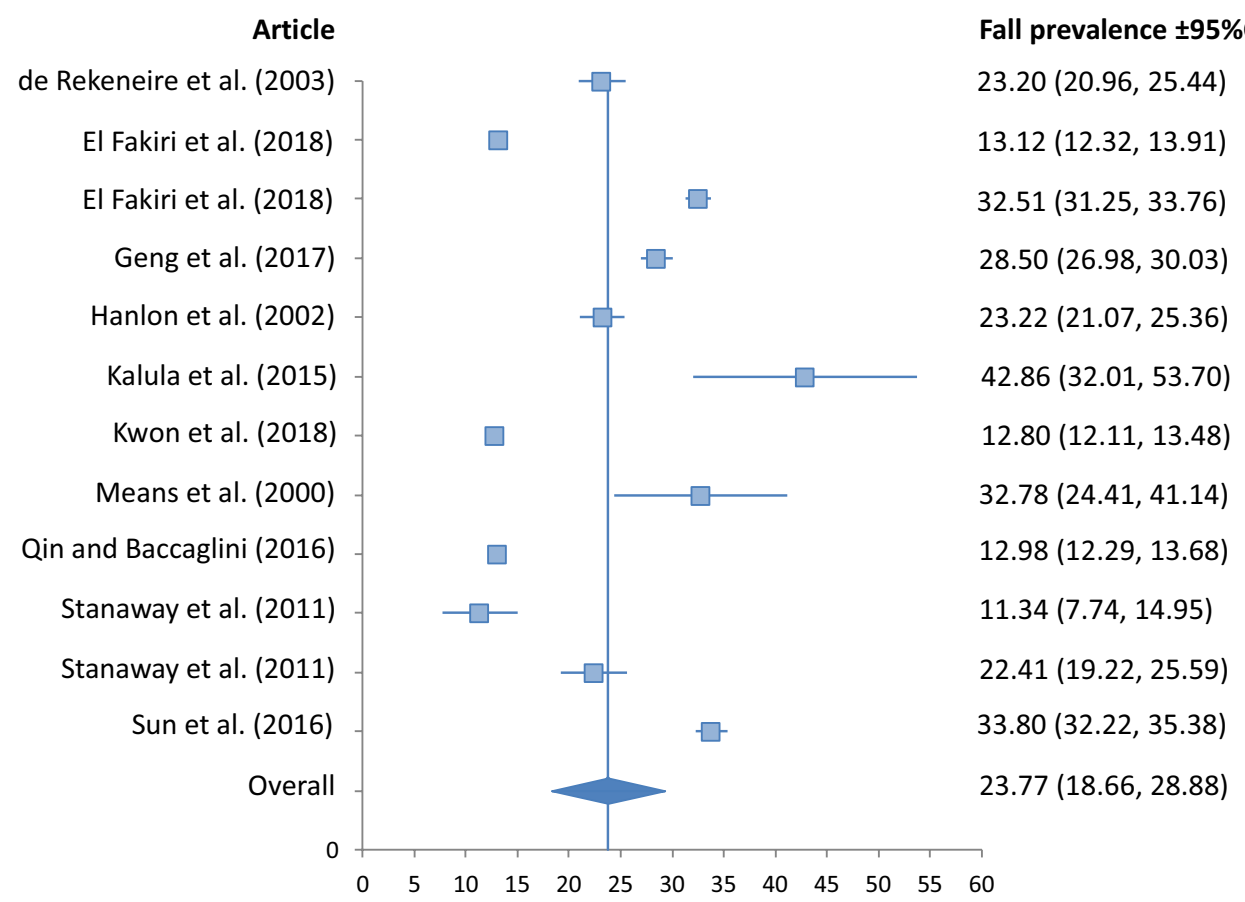

show altered gait patterns, and postural instabilities that make it difficult to recover from a perturbation [55]. The most important sociodemographic elements for falls are sex and age $[56,57]$. All these issues have considerable impacts on fall prevalence and may influence the results either by directly causing differences in the prevalence of falls, or by contributing to differences in how falls are perceived and reported by members of different ethnic groups.

The variability in this study was its main limitation. Heterogeneity was quite high, limiting general conclusions, but this is not surprising given factors such as the disparities within the general groups used. For example, the group termed Asian included Japanese, Chinese, Filipino, and 'Asian'. These nationalities are all inherently very different, with differences in all the individual factors discussed above as contributing to differences in fall prevalence.

The studies included were carried out in different countries, and with varying methodologies, which naturally cause variance. For example, study design included retrospective data, prospective data and EMR data. Most studies used a retrospective design of between 12 and 24 months. However, older adults 
Table 4 Unadjusted odds ratios/ relative risk

\begin{tabular}{|c|c|c|c|c|}
\hline Authors & Ethnic group & Gender & Sample size & Effect size \\
\hline \multirow[t]{4}{*}{ Aoyagi et al. (1998) [27] } & Japanese (Japan) & Male & 624 & - \\
\hline & Japanese (Hawaii) & Male & 436 & $1.1(0.7,1.6)$ \\
\hline & Japanese (Japan) & Female & 910 & - \\
\hline & Japanese (Hawaii) & Female & 618 & $0.8(0.6,1.1)$ \\
\hline \multirow[t]{3}{*}{ Chan et al. (2017) [28] } & Chinese (Singapore) & Male \& Female & 333 & - \\
\hline & Malay (Singapore) & Male \& Female & 31 & $2.66(1.21,5.86)^{*}$ \\
\hline & Indian (Singapore) & Male \& Female & 24 & $0.21(0.03,1.59)$ \\
\hline \multirow[t]{3}{*}{ Chen et al. (2018) [29] } & Chinese (Singapore) & Male \& Female & 1446 & - \\
\hline & Malay (Singapore) & Male \& Female & 327 & $1.45(1.05,2.00)^{*}$ \\
\hline & Indian (Singapore) & Male \& Female & 202 & $2.01(1.40,2.88)^{*}$ \\
\hline \multirow[t]{2}{*}{ Davis et al. (1999) [30] } & Japanese (Hawaii) & Female & 690 & - \\
\hline & White (USA) & Female & 9689 & $1.8(1.6,2.0)^{*}$ \\
\hline \multirow[t]{2}{*}{ Faulkner et al. (2005) [33] } & White (USA) & Female & 1665 & - \\
\hline & Black (USA) & Female & 156 & $1.17(0.78,1.75) \S$ \\
\hline \multirow[t]{4}{*}{ Geng et al. (2017) [34] } & White (USA) & Female & 4705 & - \\
\hline & Hispanic (USA) & Female & 425 & $0.97(0.74,1.27)$ \\
\hline & Black (USA) & Female & 463 & $0.77(0.59,1.00)$ \\
\hline & Asian (USA) & Female & 684 & $0.63(0.50,0.80)^{*}$ \\
\hline \multirow[t]{2}{*}{ Hanlon et al. (2002) [35] } & White (USA) & Male \& Female & 1947 & - \\
\hline & Black (USA) & Male \& Female & 1049 & $0.77(0.62,0.94)^{*}$ \\
\hline \multirow[t]{2}{*}{ Kalula et al. (2015) [36] } & Black (South Africa) & Male \& Female & 283 & - \\
\hline & White (South Africa) & Male \& Female & 140 & $1.04(1.01,1.08)^{*}$ \\
\hline \multirow[t]{5}{*}{ Karter et al. (2015) [37] } & White (USA) & Male \& Female & 63,509 & - \\
\hline & Black (USA) & Male \& Female & 11,417 & $0.64(0.59,0.70) \S^{*}$ \\
\hline & Asian (USA) & Male \& Female & 11,275 & $0.65(0.59,0.71) \S^{*}$ \\
\hline & Filipino (USA) & Male \& Female & 8162 & $0.49(0.44,0.56) \S *$ \\
\hline & Hispanic (USA) & Male \& Female & 14,324 & $0.84(0.78,0.90) \S^{*}$ \\
\hline \multirow[t]{4}{*}{ Kwan et al. (2013) [38] } & White (Austalia) & Male \& Female & 764 & - \\
\hline & Chinese (Taiwan) & Male \& Female & 280 & $0.39(0.3,0.49) \S^{*}$ \\
\hline & Chinese (Hong Kong) & Male \& Female & 201 & $0.28(0.19,0.41) \S^{*}$ \\
\hline & Chinese (Australia) & Male \& Female & 211 & $0.5(0.37,0.67) \S^{*}$ \\
\hline \multirow[t]{2}{*}{ Sun et al. (2016) [46] } & White (USA) & Male \& Female & 5186 & - \\
\hline & Black (USA) & Male \& Female & 1662 & $0.7(0.6,0.8) \S^{*}$ \\
\hline \multirow[t]{4}{*}{ Vieira et al. (2015) [47] } & Afro-Caribbean (USA) & Male \& Female & 222 & - \\
\hline & White (USA) & Male \& Female & 109 & $1.57(1.08,2.29) \S^{*}$ \\
\hline & African-American (USA) & Male \& Female & 106 & $1.63(1.07,2.47) \S *$ \\
\hline & Hispanic (USA) & Male \& Female & 113 & $1.62(1.07,2.44) \S^{*}$ \\
\hline \multirow[t]{4}{*}{ Yeong et al. (2016) [48] } & Malay (Malaysia) & Male \& Female & 631 & - \\
\hline & Chinese (Malaysia) & Male \& Female & 140 & $0.68(0.24,1.99)$ \\
\hline & Indian (Malaysia) & Male \& Female & 28 & $0.86(0.11,6.59)$ \\
\hline & Indigenous (Malaysia) & Male \& Female & 12 & $4.65(0.97,22.33)$ \\
\hline
\end{tabular}

Results are listed as Odds Ratio unless specified, § Relative Risk

$*$ significantly different from reference group $(p<0.05)$ frequently have difficulty remembering falls, whether due to having forgotten the fall, or a denial of the fall due to a desire to hide signs of frailty [58-60]. Recall of falls is generally better if the fall was serious and the person suffered a significant injury $[58,60]$, but if the injuries were minor, they too are easily forgotten
[59]. Therefore, data gathered retrospectively may not be reliable.

The sample sizes used in the different studies also varied greatly. From studies using EMR data of 5,510,341 individuals [45], to small studies containing only 114 [43]. These extremes could have very different effects 
Table 5 Adjusted odds ratios/relative risk (single falls)

\begin{tabular}{|c|c|c|c|c|c|}
\hline Authors & Ethnic group & Gender & Sample size & Effect size & Covariates \\
\hline \multirow[t]{3}{*}{ Chen et al. (2018) [29] } & Chinese (Singapore) & Male \& Female & 1446 & - & \multirow{3}{*}{$\begin{array}{l}\text { Age, sex, marrital status, cognitive } \\
\text { function, self-reported pain, } \\
\text { comorbidities, depression, BMI, } \\
\text { difficulties with ADL, social } \\
\text { network, mobility difficulties, grip } \\
\text { strength }\end{array}$} \\
\hline & Malay (Singapore) & Male \& Female & 327 & $4.76(1.21,18.68)^{*}$ & \\
\hline & Indian (Singapore) & Male \& Female & 202 & $4.50(0.73,27.64)$ & \\
\hline \multirow[t]{2}{*}{ Davis et al. (1999) [30] } & Japanese (Hawaii) & Female & 690 & - & \multirow{2}{*}{$\begin{array}{l}\text { Age, height, weight, functional } \\
\text { tests }\end{array}$} \\
\hline & White (USA) & Female & 9689 & $1.8(1.5,2.1)^{*}$ & \\
\hline \multirow[t]{2}{*}{ de Rekeneire et al. (2003) [31] } & Black (USA) & Male \& Female & 1270 & - & \multirow[t]{2}{*}{ Age, race, study site, BMI } \\
\hline & White (USA) & Male \& Female & 1780 & $1.4(1.2,1.6)^{*}$ & \\
\hline \multirow[t]{2}{*}{ Faulkner et al. (2005) [33] } & White (USA) & Female & 1665 & - & \multirow{2}{*}{$\begin{array}{l}\text { Grip strength, number of chronic } \\
\text { conditions, and depression }\end{array}$} \\
\hline & Black (USA) & Female & 156 & $1.20(0.80,1.81) \S$ & \\
\hline \multirow[t]{4}{*}{ Geng et al. (2017) [34] } & White (USA) & Female & 4705 & - & \multirow{4}{*}{$\begin{array}{l}\text { Age, co-morbidities, poor health, } \\
\text { and mobility limitations }\end{array}$} \\
\hline & Hispanic (USA) & Female & 425 & $0.94(0.71,1.24)$ & \\
\hline & Black (USA) & Female & 463 & $0.73(0.55,0.95)^{*}$ & \\
\hline & Asian (USA) & Female & 684 & $0.64(0.5,0.81)^{*}$ & \\
\hline \multirow[t]{4}{*}{ Kwan et al. (2013) [38] } & White (Austalia) & Male \& Female & 764 & - & \multirow{4}{*}{$\begin{array}{l}\text { Age, sex, incontinence, Parkin- } \\
\text { son's, education, FES-I }\end{array}$} \\
\hline & Chinese (Taiwan) & Male \& Female & 280 & $0.98(0.45,2.11) \S$ & \\
\hline & Chinese (Hong Kong) & Male \& Female & 201 & $0.55(0.17,1.79) \S$ & \\
\hline & Chinese (Australia) & Male \& Female & 211 & $0.6(0.23,1.59) \S$ & \\
\hline \multirow[t]{3}{*}{ Nicklett and Taylor (2014) [41] } & White (USA) & Male \& Female & 8429 & - & \multirow{3}{*}{$\begin{array}{l}\text { Adjusted for sociodemographic } \\
\text { and health characteristics }\end{array}$} \\
\hline & Black (USA) & Male \& Female & 1326 & $0.65(0.53,0.80)^{*}$ & \\
\hline & Hispanic (USA) & Male \& Female & 729 & $0.91(0.69,1.20)$ & \\
\hline \multirow[t]{4}{*}{ Yeong et al. (2016) [48] } & Malay (Malaysia) & Male \& Female & 631 & - & \multirow{4}{*}{$\begin{array}{l}\text { Age, sex, total income, physical } \\
\text { activity level, living alone, num- } \\
\text { ber of co-morbidities, number of } \\
\text { medications }\end{array}$} \\
\hline & Chinese (Malaysia) & Male \& Female & 140 & $0.61(0.2,1.86)$ & \\
\hline & Indian (Malaysia) & Male \& Female & 28 & $0.77(0.1,6.16)$ & \\
\hline & Indigenous (Malaysia) & Male \& Female & 12 & $6.06(1.10,33.55)^{*}$ & \\
\hline
\end{tabular}

Results are listed as Odds Ratio unless specified otherwise; $\S$ Relative Risk, * significantly different from reference group $(p<0.05)$

Activities of daily living (ADL), Body mass index (BMI), Falls efficacy scale- International (FES-I)

Table 6 Adjusted odds ratios (recurrent falls)

\begin{tabular}{|c|c|c|c|c|c|}
\hline Authors & Ethnic group & Gender & Sample size & Effect size & Covariates \\
\hline El Fakiri et al. (2018) [32] & $\begin{array}{l}\text { White (Netherlands) } \\
\text { Moroccan (Netherlands) } \\
\text { Turkish (Netherlands) } \\
\text { Surinamese (Netherlands) }\end{array}$ & $\begin{array}{l}\text { Male \& Female } \\
\text { Male \& Female } \\
\text { Male \& Female } \\
\text { Male \& Female }\end{array}$ & $\begin{array}{l}7952 \\
165 \\
188 \\
587\end{array}$ & $\begin{array}{l}- \\
0.54(0.27,1.06) \\
0.84(0.42,1.64) \\
1.05(0.68,1.64)\end{array}$ & $\begin{array}{l}\text { Age, sex, education, income, } \\
\text { deprived neighbourhood, living } \\
\text { alone, health (overweight, inactiv- } \\
\text { ity, alcohol, perecived health, } \\
\text { hearing, sight, mobility limita- } \\
\text { tions, multi-morbidity, loneliness, } \\
\text { depression) }\end{array}$ \\
\hline Kwon et al. (2018) & $\begin{array}{l}\text { White (USA) } \\
\text { Black (USA) } \\
\text { Asian (USA) } \\
\text { Hispanic (USA) }\end{array}$ & $\begin{array}{l}\text { Male \& Female } \\
\text { Male \& Female } \\
\text { Male \& Female } \\
\text { Male \& Female }\end{array}$ & $\begin{array}{l}10,527 \\
595 \\
1199 \\
1423\end{array}$ & $\begin{array}{l}- \\
0.82(0.51,1.30) \\
0.63(0.43,0.92)^{*} \\
0.98(0.72,1.34)\end{array}$ & $\begin{array}{l}\text { Age, sex, marital status, poverty, } \\
\text { BMI, chronic diseases, } \\
\text { functional limitation }\end{array}$ \\
\hline
\end{tabular}

Results are listed as Odds Ratio, * significantly different from reference group $(p<0.05)$

Body mass index (BMI) 
on the results of individual studies, with smaller sample sizes failing to identify relevant effects, and larger ones finding significant differences that are insubstantial. However, the use of a meta-analysis in this paper allowed a single estimate to be obtained for each ethnic group. Even though the larger studies using survey or EMR data were not included in the meta-analysis, the largest study in this analysis with 17,784 individuals [32], still differed greatly from the smallest indicated above.

The covariates used to adjust the data also showed considerable variation. Some studies only adjusted for basic variables such as age, race, study site and body mass index [31], while others adjusted for numerous factors such as age, gender, education, income, neighbourhood deprivation, living alone, health (being overweight, inactivity, alcohol consumption, perceived health, hearing, sight, mobility limitations, multi-morbidity, loneliness, depression) [32]. Studies in which more covariates are adjusted for increases the validity of the findings where any differences in fall prevalence between ethnicities remain. The studies in this paper showed that differences in ethnic groups remained even when ten or more covariates were included in the analysis, showing that there are differences in fall rates due to ethnicity.

The key finding of this study is that fall prevalence differs between ethnic groups, even after adjusting for multiple covariates, which underlines the importance of moving away from a 'one size fits all' approach to Public Health. Falling is a significant issue for older adults which carries considerable cost on both the personal and financial front. By identifying the most at-risk groups, resources can be targeted to where they are most needed, such as providing education and fall prevention interventions to those identified as being at risk of falls, ideally before a fall occurs. By appreciating racial and ethnic differences in fall prevalence, there can also be an equal appreciation of the different barriers and requirements of fall prevention interventions for different ethnic groups. The proposal of more ethnically tailored responses to these public health challenges may provide the answer to the low adherence of certain groups to interventions involving physical activity. Further research is needed to indicate exactly how fall prevention interventions could be better tailored to the needs of different ethnic groups, particularly in multicultural societies.

\section{Conclusion}

Differences in fall prevalence do appear to exist between different ethnic groups. Further research is required to determine the reasons for these differences, and to increase the amount of information available on fall rates of different ethnic groups.

Author Contribution The idea for this article was conceived by $\mathrm{N}$. Wehner-Hewson, the literature search and data analysis were carried out by N. Wehner-Hewson and D. Hewson, and the drafts of the article, and its critical revisions were the joint work of all authors.

Funding N. Wehner-Hewson received funding for this study from The Graduate School at the University of East London as part of an ongoing $\mathrm{PhD}$ project. The other authors have no relevant financial interests to disclose.

Data Availability Not applicable.

Code Availability Not applicable.

\section{Declarations}

Ethics Approval Consent to Participate Not applicable.

Consent for Publication Not applicable.

Conflict of Interest The authors declared no conflict of interest.

Open Access This article is licensed under a Creative Commons Attribution 4.0 International License, which permits use, sharing, adaptation, distribution and reproduction in any medium or format, as long as you give appropriate credit to the original author(s) and the source, provide a link to the Creative Commons licence, and indicate if changes were made. The images or other third party material in this article are included in the article's Creative Commons licence, unless indicated otherwise in a credit line to the material. If material is not included in the article's Creative Commons licence and your intended use is not permitted by statutory regulation or exceeds the permitted use, you will need to obtain permission directly from the copyright holder. To view a copy of this licence, visit http://creativecommons.org/licenses/by/4.0/.

\section{References}

1. James SL, Lucchesi LR, Bisignano C, Castle CD, Dingels $\mathrm{ZV}$, Fox JT, et al. The global burden of falls: global, regional and national estimates of morbidity and mortality from the Global Burden of Disease Study 2017. Injury Prevention. 2020:injuryprev-2019-043286. https://doi.org/10.1136/injur yprev-2019-043286.

2. Gill TM, Murphy TE, Gahbauer EA, Allore HG. Association of injurious falls with disability outcomes and nursing home admissions in community-living older persons. Am J Epidemiol. 2013;178(3):418-25. https://doi.org/10.1093/aje/kws554.

3. Khow KSF, Visvanathan R. Falls in the aging population. Clin Geriatr Med. 2017;33(3):357-68. https://doi.org/10.1016/j.cger. 2017.03.002.

4. Scuffham P, Chaplin S, Legood R. Incidence and costs of unintentional falls in older people in the United Kingdom. J Epidemiol Community Health. 2003;57(9):740-4. https://doi.org/10.1136/ jech.57.9.740.

5. Salkeld G, Cameron ID, Cumming RG, Easter S, Seymour J, Kurrle SE, et al. Quality of life related to fear of falling and hip fracture in older women: a time trade off study. BMJ. 
2000;320(7231):341-5. https://doi.org/10.1136/bmj.320.7231. 341.

6. Deandrea S, Lucenteforte E, Bravi F, Foschi R, La Vecchia C, Negri E. Risk factors for Falls in community-dwelling older people a systematic review and meta-analysis. Epidemiology. 2010;21(5):658-68. https://doi.org/10.1097/EDE.0b013e3181 e89905.

7. Patel N, Ferrer HB, Tyrer F, Wray P, Farooqi A, Davies MJ, et al. Barriers and facilitators to healthy lifestyle changes in minority ethnic populations in the UK: a narrative review. J Racial Ethn Health Disparities. 2017;4(6):1107-19. https://doi.org/10.1007/ s40615-016-0316-y.

8. Bhopal R. Chronic diseases in Europes migrant and ethnic minorities: challenges, solutions and a vision. Eur J Pub Health. 2009;19(2):140-3. https://doi.org/10.1093/eurpub/ckp024.

9. Evandrou M, Falkingham J, Feng Z, Vlachantoni A. Ethnic inequalities in limiting health and self-reported health in later life revisited. J Epidemiol Community Health. 2016;70(7):653-62. https://doi.org/10.1136/jech-2015-206074.

10. Hui A, Latif A, Hinsliff-Smith K, Chen T. Exploring the impacts of organisational structure, policy and practice on the health inequalities of marginalised communities: Illustrative cases from the UK healthcare system. Health Policy (Amsterdam, Netherlands). 2020;124(3):298-302. https://doi.org/10.1016/j.healthpol.2020. 01.003.

11. Sproston K, Mindell J. Health Survey for England 2004. The health of minority ethnic groups. NHS Health and Social Care Information Centre, Public Health Statistics. 2006.

12. World Health Organization. World report on ageing and health. World Health Organization; 2015.

13. Clegg A, Young J, Iliffe S, Rikkert MO, Rockwood K. Frailty in elderly people. Lancet. 2013;381(9868):752-62. https://doi.org/ 10.1016/s0140-6736(12)62167-9.

14. Brothers TD, Theou O, Rockwood K. Frailty and migration in middle-aged and older Europeans. Arch Gerontol Geriatr. 2014;58(1):63-8. https://doi.org/10.1016/j.archger.2013.07.008.

15. Herd P, Robert SA, House JS. Health disparities among older adults: Life course influences and policy solutions. Handbook of aging and the social sciences. Elsevier; 2011. p. 121-34.

16. Gale CR, Cooper C, Sayer AA. Prevalence of frailty and disability: findings from the English longitudinal study of ageing. Age Ageing. 2015;44(1):162-5. https://doi.org/10.1093/ageing/afu148.

17. Tinetti ME, Speechley M, Ginter SF. Risk-factors for falls among elderly persons living in the communnity. N Engl J Med. 1988;319(26):1701-7. https://doi.org/10.1056/nejm19881229319 2604.

18. Kwan MMS, Close JCT, Wong AKW, Lord SR. Falls incidence, risk factors, and consequences in Chinese older people: a systematic review. J Am Geriatr Soc. 2011;59(3):536-43. https://doi.org/ 10.1111/j.1532-5415.2010.03286.x.

19. Blankinship LA, Rouse WA, Bernstein J, Kruk J, Aboul-Enein BH. A narrative review of ethnic minority studies for faith-based health promotion interventions with special reference to the contemporary Christian nurse. J Relig Health. 2021;60(2):1375-87. https://doi.org/10.1007/s10943-020-01150-0.

20. Cela E, Barbiano di Belgiojoso E. Ageing in a foreign country: determinants of self-rated health among older migrants in Italy. $\mathbf{J}$ Ethn Migr Stud. 2019:1-23.

21. Liberati A, Altman DG, Tetzlaff J, Mulrow C, Gotzsche PC, Ioannidis JPA, et al. The PRISMA statement for reporting systematic reviews and meta-analyses of studies that evaluate health care interventions: explanation and elaboration. Plos Medicine. 2009;6(7). https://doi.org/10.1371/journal.pmed.1000100.

22. Lockwood C, Munn Z, Porritt K. Qualitative research synthesis: methodological guidance for systematic reviewers utilizing meta-aggregation. Int J Evid Based Healthc. 2015;13(3):179-87. https://doi.org/10.1097/xeb.0000000000000062.

23. National Heart Lung and Blood Institute: Development and use of quality assessment tools. www.nhlbi.nih.gov/health-topics/studyquality-assessment-tools. Accessed 16 Apr 2021.

24. National Institutes of Health. NOT-OD-15-089: racial and ethnic categories and definitions for NIH diversity programs and for other reporting purposes. Bethesda: NIH; 2015.

25. Higgins JPT, Thompson SG, Deeks JJ, Altman DG. Measuring inconsistency in meta-analyses. BMJ. 2003;327(7414):557-60. https://doi.org/10.1136/bmj.327.7414.557.

26. Neyeloff JL, Fuchs SC, Moreira LB. Meta-analyses and Forest plots using a microsoft excel spreadsheet: step-by-step guide focusing on descriptive data analysis. BMC Res Notes. 2012;5:52. https://doi.org/10.1186/1756-0500-5-52.

27. Aoyagi K, Ross PD, Davis JW, Wasnich RD, Hayashi T, Takemoto T. Falls among community-dwelling elderly in Japan. J Bone Miner Res. 1998;13(9):1468-74. https://doi.org/10.1359/ jbmr.1998.13.9.1468.

28. Chan KM, Pang WS, Ee CH, Ding YY, Choo P. Epidemiology of falls among the elderly community dwellers in Singapore. Singapore Med J. 1997;38(10):427-31.

29. Chen TY, Tan PJ, Chan A. Ethnicity predicts falls among community-dwelling older adults in Singapore. Geriatr Gerontol Int. 2018;18(1):72-9. https://doi.org/10.1111/ggi.13143.

30. Davis JW, Nevitt MC, Wasnich RD, Ross PD. A cross-cultural comparison of neuromuscular performance, functional status, and falls between Japanese and white women. J Gerontology A Biol Sci Med Sci. 1999;54(6):M288-92. https://doi.org/10. 1093/gerona/54.6.M288.

31. de Rekeneire N, Visser M, Peila R, Nevitt MC, Cauley JA, Tylavsky FA, et al. Is a fall just a fall: Correlates of falling in healthy older persons. The health, aging and body composition study. J Am Geriatr Soc. 2003;51(6):841-6. https://doi.org/10. 1046/j.1365-2389.2003.51267.x.

32. El Fakiri F, Kegel AA, Schouten GM, Berns MPH. Ethnic differences in fall risk among community-dwelling older people in the Netherlands. J Aging Health. 2018;30(3):365-85. https:// doi.org/10.1177/0898264316679531.

33. Faulkner KA, Cauley JA, Zmuda JM, Landsittel DP, Nevitt MC, Newman AB, et al. Ethnic differences in the frequency and circumstances of falling in older community-dwelling women. J Am Geriatr Soc. 2005;53(10):1774-9. https://doi.org/10.1111/j. 1532-5415.2005.53514.x.

34. Geng YF, Lo JC, Brickner L, Gordon NP. Racial-ethnic differences in fall prevalence among older women: a cross-sectional survey study. Bmc Geriatrics. 2017;17:65. https://doi.org/10. 1186/s12877-017-0447-y.

35. Hanlon JT, Landerman LR, Fillenbaum GG, Studenski S. Falls in African American and white community-dwelling elderly residents. J Gerontol A Biol Sci Med Sci. 2002;57(7):M473-8. https://doi.org/10.1093/gerona/57.7.M473.

36. Kalula SZ, Ferreira M, Swingler GH, Badri M. Ethnic differences in rates and causes of falls in an urban communitydwelling older population in South Africa. J Am Geriatr Soc. 2015;63(2):403-4. https://doi.org/10.1111/jgs.13277.

37. Karter AJ, Laiteerapong N, Chin MH, Moffet HH, Parker MM, Sudore R, et al. Ethnic differences in geriatric conditions and diabetes complications among older, insured adults with diabetes: the diabetes and aging study. J Aging Health. 2015;27(5):894-918. https://doi.org/10.1177/0898264315 569455 .

38. Kwan MMS, Tsang WWN, Lin SI, Greenaway M, Close JCT, Lord SR. Increased concern is protective for falls in Chinese older people: the chopstix fall risk study. J Gerontol A Biol Sci Med Sci. 2013;68(8):946-53. https://doi.org/10.1093/gerona/gls338. 
39. Leong Joyce WS, Zukri INM, Ching SM, Devaraj NK. Factors associated with falls among the elderly attending a government clinic in Kuala Lumpur. Malays J Med Health Sci. 2020;16(1):183-90.

40. Means KM, O'Sullivan PS, Rodell DE. Balance, mobility, and falls among elderly African American women. Am J Phys Med Rehabil. 2000;79(1):30-9. https://doi.org/10.1097/00002060200001000-00008.

41. Nicklett EJ, Taylor RJ. Racial/ethnic predictors of falls among older adults: the health and retirement study. J Aging Health. 2014;26(6):1060-75. https://doi.org/10.1177/0898264314541698.

42. Qin ZJ, Baccaglini L. Distribution, determinants, and prevention of falls among the elderly in the 2011-2012 California Health Interview Survey. Public Health Rep. 2016;131(2):331-9. https:// doi.org/10.1177/003335491613100217.

43. Sampaio RAC, Sewo Sampaio PY, Yamada M, Ogita M, Matsudo SMM, Raso V, et al. Factors associated with falls in active older adults in Japan and Brazil. J Clin Gerontol Geriatr. 2013;4(3):89_ 92. https://doi.org/10.1016/j.jcgg.2013.04.003.

44. Stanaway FF, Cumming RG, Naganathan V, Blyth FM, Handelsman DJ, Le Couteur DG, et al. Ethnicity and falls in older men: low rate of falls in Italian-born men in Australia. Age Ageing. 2011;40(5):595-601. https://doi.org/10.1093/ageing/afr067.

45. Stevens JA, Mack KA, Paulozzi LJ, Ballesteros MF. Self-reported falls and fall-related injuries among persons aged $>=65$ years United States, 2006. J Safety Res. 2008;39(3):345-9. https://doi. org/10.1016/j.jsr.2008.05.002.

46. Sun DQ, Huang J, Varadhan R, Agrawal Y. Race and fall risk: data from the National Health and Aging Trends Study (NHATS). Age Ageing. 2016;45(1):120-7. https://doi.org/10.1093/ageing/afv173.

47. Vieira ER, Tappen R, Engstrom G, da Costa BR. Rates and factors associated with falls in older European Americans, Afro-Caribbeans, African-Americans, and Hispanics. Clin Interv Aging. 2015;10:1705-10. https://doi.org/10.2147/cia.s91120.

48. Yeong UY, Tan SY, Yap JF, Choo WY. Prevalence of falls among community-dwelling elderly and its associated factors: a crosssectional study in Perak, Malaysia. Malays Fam Physician. 2016;11(1):7-14.

49. Cunningham TJ, Croft JB, Liu Y, Lu H, Eke PI, Giles WH. Vital signs: racial disparities in age-specific mortality among Blacks or African Americans - United States, 1999-2015. MMWR Morbidity and mortality weekly report. 2017;66(17):444-56. https://doi. org/10.15585/mmwr.mm6617e1.

50. Caldwell JT, Lee H, Cagney KA. Disablement in context: neighborhood characteristics and their association with frailty onset among older adults. J Gerontol B Psychol Sci Soc Sci. 2019;74(7):E40-9. https://doi.org/10.1093/geronb/gbx123.
51. Hirsch C, Anderson ML, Newman A, Kop W, Jackson S, Gottdiener J, et al. The association of race with frailty: the cardiovascular health study. Ann Epidemiol. 2006;16(7):545-53. https://doi. org/10.1016/j.annepidem.2005.10.003.

52. Huang HC, Tsai TF, Subeq YM. Using grey relational analysis and grey integrated multi-objective strategy to evaluate the risk factors of falling of aboriginal elders in Taiwan. Soft Comput. 2020;24(11):8097-112. https://doi.org/10.1007/ s00500-019-04178-x

53. Horne M, Speed S, Skelton D, Todd C. What do communitydwelling Caucasian and South Asian 60-70 year olds think about exercise for fall prevention ? Age Ageing. 2009;38(1):68-73. https://doi.org/10.1093/ageing/afn237.

54. Horton K, Dickinson A. The role of culture and diversity in the prevention of falls among older Chinese people. Can J AgingRevue Canadienne Du Vieillissement. 2011;30(1):57-66. https:// doi.org/10.1017/s0714980810000826.

55. Lockhart TE, Frames CW, Soangra R, Lieberman A. Effects of obesity and fall risk on gait and posture of community-dwelling older adults. Int J Progn Health Manag. 2019;10(1):019.

56. Gale CR, Cooper C, Sayer AA. Prevalence and risk factors for falls in older men and women: the English longitudinal study of ageing. Age Ageing. 2016;45(6):789-94. https://doi.org/10.1093/ ageing/afw129.

57. Chang VC, Do MT. Risk factors for falls among seniors: implications of gender. Am J Epidemiol. 2015;181(7):521-31. https://doi. org/10.1093/aje/kwu268.

58. Garcia PA, Dias JMD, Silva SLA, Dias RC. Prospective monitoring and self-report of previous falls among older women at high risk of falls and fractures: a study of comparison and agreement. Braz J Phys Ther. 2015;19(3):218-26. https://doi.org/10.1590/ bjpt-rbf.2014.0095.

59. Cummings SR, Nevitt MC, Kidd S. Forgetting falls- the limited accuracy of recall of falls in the elderly. J Am Geriatr Soc. 1988;36(7):613-6. https://doi.org/10.1111/j.1532-5415.1988. tb06155.x.

60. Peel N. Validating recall of falls by older people. Accid Anal Prev. 2000;32(3):371-2. https://doi.org/10.1016/s0001-4575(99) 00066-4.

Publisher's Note Springer Nature remains neutral with regard to jurisdictional claims in published maps and institutional affiliations. 